\title{
Hypoglycaemia following diabetes remission in patients with 6q24 methylation defects: expanding the clinical phenotype
}

\author{
S. E. Flanagan • D. J. G. Mackay • S. A. W. Greeley • \\ T. J. McDonald • V. Mericq • J. Hassing • \\ E. J. Richmond • W. R. Martin • C. Acerini • \\ A. M. Kaulfers • D. P. Flynn - J. Popovic • \\ M. A. Sperling • K. Hussain • S. Ellard • A. T. Hattersley
}

Received: 3 August 2012 / Accepted: 12 October 2012 / Published online: 31 October 2012

(C) Springer-Verlag Berlin Heidelberg 2012

Keywords Chromosome 6q24 · Hypoglycaemia · Transient neonatal diabetes mellitus $\cdot$ Uniparental disomy

\section{Abbreviations \\ TNDM Transient neonatal diabetes mellitus \\ UPD Uniparental disomy}

To the Editor: Methylation defects at chromosome 6q24 are the most common cause of transient neonatal diabetes

S. E. Flanagan $(\bowtie) \cdot$ T. J. McDonald $\cdot$ S. Ellard $\cdot$ A. T. Hattersley University of Exeter Medical School,

Barrack Road,

Exeter, Devon EX2 5DW, UK

e-mail: sarah.flanagan@pms.ac.uk

D. J. G. Mackay

Wessex Regional Genetics Laboratory, Salisbury District Hospital, Salisbury, UK

\section{S. A. W. Greeley}

Kovler Diabetes Center, The University of Chicago,

Chicago, IL, USA

\section{Mericq}

Institute of Maternal and Child Research, Faculty of Medicine, University of Chile,

Santiago, Chile

J. Hassing

Pediatric Endocrinology, Rockwood Clinic,

Spokane, WA, USA

\section{E. J. Richmond}

Pediatric Endocrinology, National Children's Hospital,

San José, Costa Rica mellitus (TNDM), accounting for $70 \%$ of all cases $[1,2]$. Those affected have impaired insulin secretion, as shown by a very low birthweight (median below the first centile) and a diagnosis of diabetes usually at or shortly after birth (range 0-4 weeks) [1-3]. In most cases the diabetes remits by a median age of 13 weeks, although many will experience a relapse later in life [3]. Loss of methylation at the chromosome 6q24 locus results from one of three mechanisms: (1) paternal uniparental disomy (UPD) (approximately $40 \%$ of cases); (2) paternal duplication (approximately $32 \%$ of

W. R. Martin

Pediatric Endocrinology, Sacred Heart Children's Hospital, Spokane, WA, USA

C. Acerini

Department of Paediatrics, University of Cambridge,

Cambridge, UK

A. M. Kaulfers

Pediatric Endocrinology, University of South Alabama, Mobile, AL, USA

D. P. Flynn · J. Popovic $\cdot$ M. A. Sperling

Division of Pediatric Endocrinology, Children's Hospital of Pittsburgh of UPMC, University of Pittsburgh School of Medicine, Pittsburgh, PA, USA

K. Hussain

Department of Endocrinology,

Great Ormond Street Hospital for Children NHS Trust,

London, UK

K. Hussain

The Institute of Child Health, University College London,

London, UK 
cases); or (3) loss of methylation without a structural chromosome defect (approximately $28 \%$ of cases) (reviewed in [2]). The TNDM critical region on chromosome $6 \mathrm{q} 24$ encompasses PLAGL1, a tumour-suppressor gene, and HYMAI, a noncoding RNA of unknown function [2]. The underlying mechanism(s) by which loss of methylation, and hence overexpression, of PLAGL1 and/or HYMAI cause TNDM is not known.

We report the novel clinical observation of hypoglycaemia following diabetes remission in six out of 43 patients (14\%) with 6q24 TNDM. This is in keeping with the prevalence of extra-pancreatic features of patients with $6 \mathrm{q} 24$ TNDM, such as macroglossia (35\%) and umbilical hernia $(14 \%)$ [2]. Five of the six patients had paternal UPD and one patient had a paternal duplication [4]. No differences in diabetes were observed between patients with and without hypoglycaemia, as shown by the age at diagnosis (1 week vs 4 days, $p=0.87$ ) and age at remission (18 vs 21 weeks, $p=$ 0.41 ). The median birthweight was, however, higher in the six patients with hypoglycaemia than in the 37 patients in whom hypoglycaemia was not reported $(-1.83$ vs -3.14 standard deviation score, $p=0.026$ ), which is in keeping with increased insulin secretion in utero. Inactivating $A B C C 8$ and $K C N J 11$ gene mutations, the most common cause of hyperinsulinaemic hypoglycaemia, were excluded by sequence analysis [5].

Hypoglycaemia (blood glucose $<2.6 \mathrm{mmol} / \mathrm{l}$ ) was diagnosed at a median age of 33.5 weeks, which was within 222 weeks of diabetes remission. The clinical characteristics of the six patients are shown in Table 1. The presentation of hypoglycaemia was variable. In three cases (patients 1, 5 and 6), hypoglycaemia was noted on blood measurement following hospital admission for a self-limiting viral illness. Three other patients (patients 2, 3 and 4) were symptomatic, with lethargy and shakiness that improved with feeding.

All six patients required treatment for hypoglycaemia and only one did not require long-term treatment (Table 1). Diazoxide (5-15 $\mathrm{mgkg}^{-1} \mathrm{day}^{-1}$ ) was given to four patients, with three continuing to require treatment 1,2 and 4 years later (patients 1, 3 and 4, respectively). One patient required overnight bolus feeds to prevent hypoglycaemia 2 years after diagnosis and has only recently started diazoxide therapy.

The hypoglycaemia may result from excess insulin secretion. Serum insulin was measured at the time of hypoglycaemia in five of the six patients: in three cases insulin was found to be inappropriately high. In the remaining two patients, insulin was below the assay limit of detection on a single measurement but blood ketones were inappropriately suppressed $(<0.2 \mathrm{nmol} / \mathrm{l})$ at the time of hypoglycaemia, suggesting an insulin-mediated action. Growth hormone and cortisol levels were assessed in four patients, and deficiency of these hormones was ruled out as a cause of hypoglycaemia. Further investigations are required to confirm that hyperinsulinism is the mechanism of hypoglycaemia in these patients.
The high prevalence of hypoglycaemia in our cohort (14\%) when compared with an incidence of hyperinsulinaemic hypoglycaemia of $0.002 \%$ in outbred populations means that it is very likely that the hypoglycaemia is a direct consequence of the chromosome 6q24 abnormality [6]. The mechanism of the hypoglycaemia is not currently understood.

To investigate the possibility of a recessively acting mutation unmasked by UPD, we undertook exome sequencing in three patients (patients 1, 2 and 3). We analysed all genomic regions corresponding to the NCBI Consensus Coding Sequence database captured by the Agilent SureSelect Human All Exon Kit (v1; Agilent Technologies, Santa Clara, CA, USA). Paired-end sequencing was performed on an Illumina GAII (Illumina, San Diego, CA, USA) and all variants identified in the minimum shared region of UPD $(29.9 \mathrm{~kb}$, as defined by Affymetrix Human SNP Assay 6.0 [Affymetrix, Santa Clara, CA, USA], flanking SNPs rs13220827 and rs6931065) were identified using the Genome Analysis Toolkit (http://www.broadinstitute.org/gatk/). No genes harbouring a novel non-synonymous variant were identified in more than one individual. This excludes coding mutations in the captured exons, but does not exclude a causal mutation in a non-coding or regulatory region. The presence of a paternal duplication in one patient suggests that hypoglycaemia is more likely to be a direct consequence of the methylation defect than of the chromosome abnormality causing it. However, it is noteworthy that the five patients with UPD had a more severe phenotype, as demonstrated by long-term requirements for diazoxide or overnight bolus feeds when compared with the patient with the duplication, who had episodic hypoglycaemia. Studies on larger numbers of patients are required to determine whether this observation reflects a genotype-phenotype relationship.

The reason for the remission of diabetes in patients with 6q24 TNDM is not known. Studies in mice with paternal inheritance of a transgene have shown an increase in the number of pancreatic beta cells prior to diabetes remission [7], and it is possible that in these patients there is an 'overshoot' of this process and consequently beta cell hyperplasia. However, this, on its own, cannot explain why the beta cells inappropriately secrete insulin despite hypoglycaemia. While it is known that mutations in $A B C C 8$, $K C N J 11$ and $H N F 4 A$ can cause transient congenital hyperinsulinaemic hypoglycaemia and later-onset diabetes, there have not been any reports of hyperinsulinaemic hypoglycaemia developing following diabetes remission [5, 8]. Further studies are required to establish the cause of the severe defect in the regulation of insulin secretion in these patients.

In conclusion, the identification of hypoglycaemia in $14 \%$ of patients with $6 \mathrm{q} 24$ TNDM provides further evidence for the key role of the chromosome 6q24 locus in the regulation of insulin secretion and glucose homeostasis. It is important to be aware of the increased risk of 


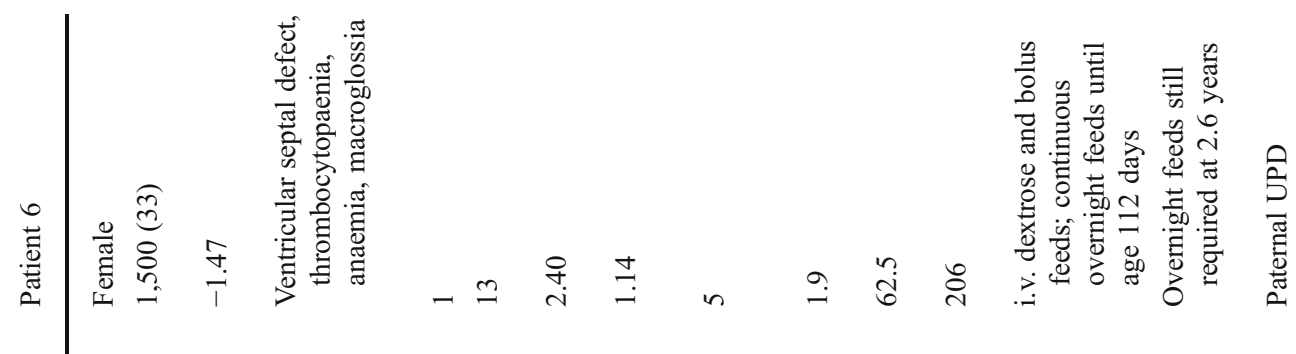

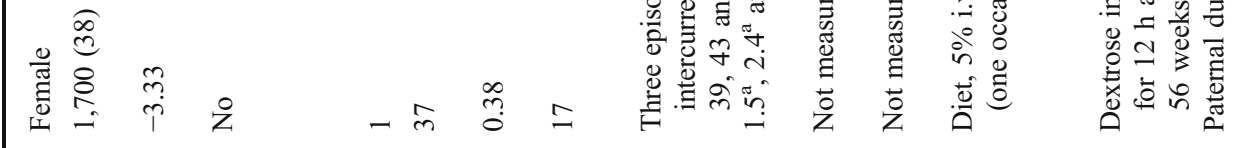

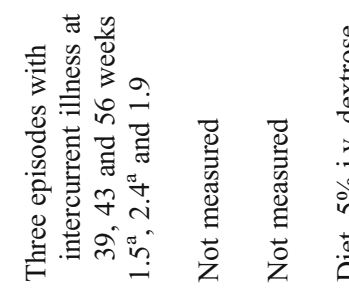

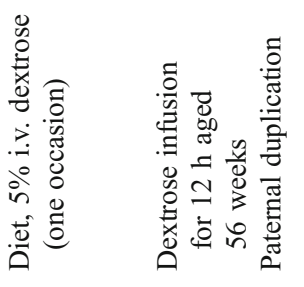
(n)

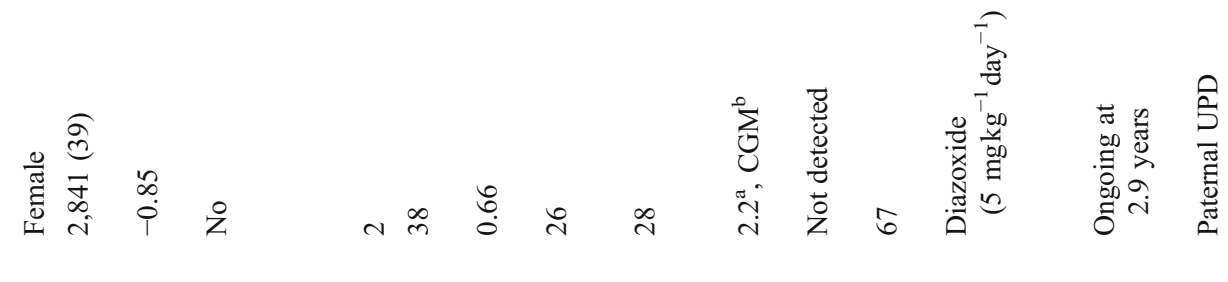

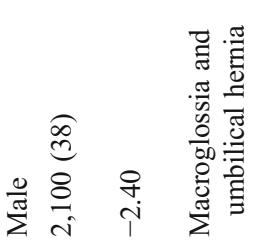
(n) 
hypoglycaemia in the months following diabetes remission in patients with a chromosome 6q24 methylation defect.

Acknowledgements S. E. Flanagan was the Sir Graham Wilkins, Peninsula Medical School Research Fellow. A. T. Hattersley and S. Ellard are employed as core members of staff within the National Institute for Health Research-funded Peninsula Clinical Research Facility.

Contribution statement SEF and DJGM performed molecular genetic testing. SEF and ATH were responsible for the conception of the study and drafted the manuscript. All authors analysed data and revised the manuscript. All authors approved the final version.

\section{References}

1. Gardner RJ, Mackay DJ, Mungall AJ et al (2000) An imprinted locus associated with transient neonatal diabetes mellitus. Hum Mol Genet 9:589-596
2. Mackay DJ, Temple IK (2010) Transient neonatal diabetes mellitus type 1. Am J Med Genet C Semin Med Genet 154C:335-342

3. Flanagan SE, Patch AM, Mackay DJ et al (2007) Mutations in ATPsensitive $\mathrm{K}^{+}$channel genes cause transient neonatal diabetes and permanent diabetes in childhood or adulthood. Diabetes 56:1930 1937

4. Mackay DJ, Temple IK, Shield JP, Robinson DO (2005) Bisulphite sequencing of the transient neonatal diabetes mellitus DMR facilitates a novel diagnostic test but reveals no methylation anomalies in patients of unknown aetiology. Hum Genet 116:255-261

5. Kapoor RR, Flanagan SE, James C, Shield J, Ellard S, Hussain K (2009) Hyperinsulinaemic hypoglycaemia. Arch Dis Child 94:450 457

6. Otonkoski T, Ammala C, Huopio H et al (1999) A point mutation inactivating the sulfonylurea receptor causes the severe form of persistent hyperinsulinemic hypoglycemia of infancy in Finland. Diabetes 48:408-415

7. Ma D, Shield JP, Dean W et al (2004) Impaired glucose homeostasis in transgenic mice expressing the human transient neonatal diabetes mellitus locus, TNDM. J Clin Invest 114:339-348

8. Pearson ER, Boj SF, Steele AM et al (2007) Macrosomia and hyperinsulinaemic hypoglycaemia in patients with heterozygous mutations in the HNF4A gene. PLoS Med 4:e118 